\title{
AMBER, the near-infrared spectro-interferometric three-telescope VLTI instrument
}

R. G. Petrov ${ }^{1}$, F. Malbet ${ }^{2}$, G. Weigelt ${ }^{3}$, P. Antonelli ${ }^{4}$, U. Beckmann $^{3}$, Y. Bresson ${ }^{4}$, A. Chelli ${ }^{2}$, M. Dugué ${ }^{4}$, G. Duvert ${ }^{2}$, S. Gennari ${ }^{5}$, L. Glück ${ }^{2}$, P. Kern ${ }^{2}$, S. Lagarde ${ }^{4}$, E. Le Coarer ${ }^{2}$, F. Lisi ${ }^{5}$, F. Millour ${ }^{1,2}$, K. Perraut ${ }^{2}$, P. Puget ${ }^{2}$, F. Rantakyrö ${ }^{6}$, S. Robbe-Dubois ${ }^{1}$, A. Roussel ${ }^{4}$, P. Salinari ${ }^{5}$, E. Tatulli2 ${ }^{2,5}$, G. Zins ${ }^{2}$, M. Accardo ${ }^{5}$, B. Acke ${ }^{2,13}$, K. Agabi ${ }^{1}$, E. Altariba ${ }^{2}$, B. Arezki ${ }^{2}$, E. Aristidi ${ }^{1}$, C. Baffa ${ }^{5}$, J. Behrend ${ }^{3}$, T. Blöcker ${ }^{3}$, S. Bonhomme ${ }^{4}$, S. Busoni ${ }^{5}$, F. Cassaing ${ }^{7}$, J.-M. Clausse ${ }^{4}$, J. Colin $^{4}$, C. Connot ${ }^{3}$, A. Delboulbé ${ }^{2}$, A. Domiciano de Souza ${ }^{1,4}$, T. Driebe ${ }^{3}$, P. Feautrier ${ }^{2}$, D. Ferruzzi ${ }^{5}$, T. Forveille ${ }^{2}$, E. Fossat ${ }^{1}$, R. Foy ${ }^{8}$, D. Fraix-Burnet ${ }^{2}$, A. Gallardo ${ }^{2}$, E. Giani ${ }^{5}$, C. Gil $^{2}, 14$, A. Glentzlin ${ }^{4}$, M. Heiden ${ }^{3}$, M. Heininger ${ }^{3}$, O. Hernandez Utrera ${ }^{2}$, K.-H. Hofmann ${ }^{3}$, D. Kamm ${ }^{4}$, M. Kiekebusch ${ }^{6}$, S. Kraus ${ }^{3}$, D. Le Contel ${ }^{4}$, J.-M. Le Contel ${ }^{4}$, T. Lesourd ${ }^{9}$, B. Lopez ${ }^{4}$, M. Lopez ${ }^{9}$, Y. Magnard ${ }^{2}$, A. Marconi ${ }^{5}$, G. Mars ${ }^{4}$, G. Martinot-Lagarde ${ }^{9,4}$, P. Mathias ${ }^{4}$, P. Mège ${ }^{2}$, J.-L. Monin ${ }^{2}$, D. Mouillet ${ }^{2,15}$, D. Mourard ${ }^{4}$, E. Nussbaum ${ }^{3}$, K. Ohnaka ${ }^{3}$, J. Pacheco ${ }^{4}$, C. Perrier ${ }^{2}$, Y. Rabbia ${ }^{4}$, S. Rebattu ${ }^{4}$, F. Reynaud ${ }^{10}$, A. Richichi ${ }^{11}$, A. Robini ${ }^{1}$, M. Sacchettini ${ }^{2}$, D. Schertl ${ }^{3}$, M. Schöller ${ }^{6}$, W. Solscheid ${ }^{3}$, A. Spang ${ }^{4}$, P. Stee ${ }^{4}$, P. Stefanini ${ }^{5}$, M. Tallon ${ }^{8}$,

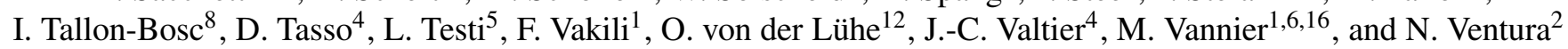

(Affiliations can be found after the references)

Received 3 October 2006 / Accepted 6 December 2006

\section{ABSTRACT}

Context. Optical long-baseline interferometry is moving a crucial step forward with the advent of general-user scientific instruments that equip large aperture and hectometric baseline facilities, such as the Very Large Telescope Interferometer (VLTI).

Aims. AMBER is one of the VLTI instruments that combines up to three beams with low, moderate and high spectral resolutions in order to provide milli-arcsecond spatial resolution for compact astrophysical sources in the near-infrared wavelength domain. Its main specifications are based on three key programs on young stellar objects, active galactic nuclei central regions, masses, and spectra of hot extra-solar planets.

Methods. These key science goals led to scientific specifications, which were used to propose and then validate the instrument concept. AMBER uses single-mode fibers to filter the entrance signal and to reach highly accurate, multiaxial three-beam combination, yielding three baselines and a closure phase, three spectral dispersive elements, and specific self-calibration procedures.

Results. The AMBER measurements yield spectrally dispersed calibrated visibilities, color-differential complex visibilities, and a closure phase allows astronomers to contemplate rudimentary imaging and highly accurate visibility and phase differential measurements. AMBER was installed in 2004 at the Paranal Observatory. We describe here the present implementation of the instrument in the configuration with which the astronomical community can access it.

Conclusions. After two years of commissioning tests and preliminary observations, AMBER has produced its first refereed publications, allowing assessment of its scientific potential.

Key words. instrumentation: high angular resolution - techniques: interferometric - techniques: spectroscopic

\section{Introduction}

Long-baseline interferometry using optical telescopes has reached an important stage in its development at the beginning of the 21 st century by combining the light of astrophysical sources collected by large apertures with the Very Large Telescope Interferometer (VLTI; Glindemann et al. 2001a,b) and the Keck Interferometer (KI; Colavita et al. 2003). This achievement allows the observers to obtain unprecedented spatial resolution, together with an enhanced flux sensitivity compared to earlier interferometers. This was first demonstrated by the extragalactic observations with the KI (Swain et al. 2003) and VLTI (Jaffe et al. 2004; Wittkowski et al. 2004).

The VLTI (Glindemann et al. 2004) is the infrastructure located on the summit of Cerro Paranal in Chile that is necessary for performing optical interferometry. It includes large 8-m telescopes called Unit Telescopes (UTs) and 2-m telescopes called
Auxiliary Telescopes (ATs), but also the optical train that allows the light beam collected by the apertures to be conveyed to the combining instrument. Each beam is partially corrected for atmospheric wave front perturbation thanks to adaptive optics (AO) modules on UTs or to tip-tilt correctors for ATs. The beams are transported to the interferometric laboratory through the telescope Coudé trains feeding delay lines (DLs) installed in a thermally stable interferometric tunnel. As a first step, the optical path difference (OPD) between two beams is set to zero with errors smaller than $100 \mu \mathrm{m}$ thanks to the good global metrology of the instrument. A fringe sensor (Gai et al. 2004) is being implemented in the OPD control loop to stabilize the fringes within a small fraction of wavelength. For each beam, the pupils and the images delivered in the focal laboratory are stabilized. Eventually, a field separator to be implemented at each telescope (Delplancke et al. 2004) will allow AO correction and fringe tracking on an off axis reference star up to 1 arc minute away 


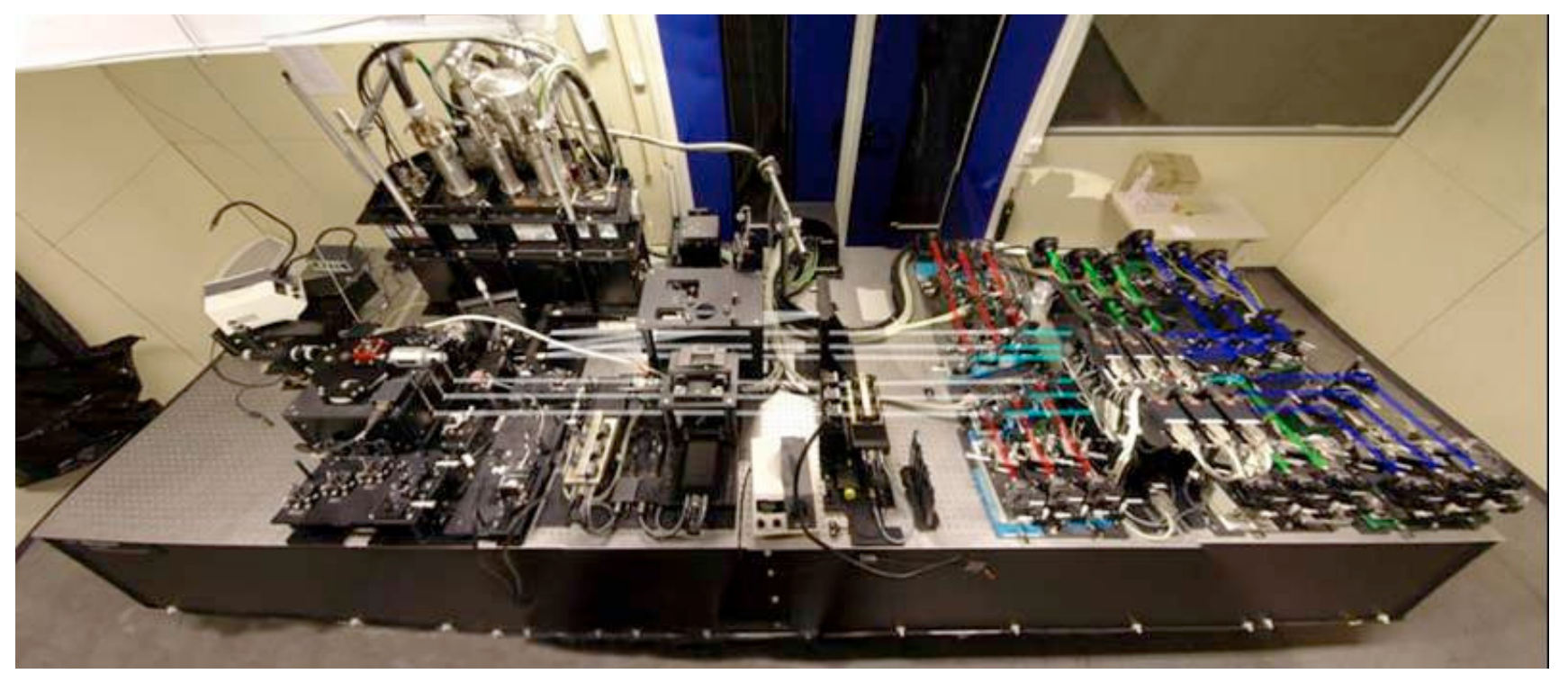

Fig. 1. Composite photograph of the AMBER instrument in the integration room of the Laboratoire d'Astrophysique de Grenoble in 2003. The instrument was complete but for its enclosure and for the beam commutation device. Some integration and test tools are also still present on the table. The beams (white lines) arrive in the bottom left of the picture and travel from the left to the right. They are split spectrally by dichroïcs in $K$, $H$, and $J$ bands (respectively red, green, and blue from left to right) before being fed into single-mode optical fibers that filter each beam spatially. After the spatial filters, the bands are merged together by a symmetrical set of dichroïcs, then travel right to left through cylindrical optics and a periscope, and is finally focused on the entrance slit of the cryogenic spectrograph in the upper left corner. The 1600 Kg AMBER table is 4.2 by $1.5 \mathrm{~m}$ and supports about $300 \mathrm{Kg}$ of optical and mechanical equipment.

from the scientific source. The ultimate feature of the VLTI will be a metrology system combined with differential delay lines allowing us to perform imaging through phase referencing between an off-axis reference star and the scientific source, as well as highly accurate differential astrometry (Paresce et al. 2003a). The implementation of the VLTI is progressive. It started with two telescopes without fringe tracking and will eventually reach the full picture described above.

The VLTI is completed with focal instruments that clean or calibrate some perturbations in each beam, combine two or more beams, and record and analyze the interference fringes in one or several spectral channels. VINCI (Kervella et al. 2003), a twoway beam combiner working in the broad $K$-band with a fiber coupler, has been used for commissioning the first stages of the VLTI and has produced a wealth of science (Paresce et al. 2003b) but is no longer proposed to the community. MIDI (Leinert et al. 2003; Leinert 2004) is a two-way beam combiner for the midinfrared $N$-band featuring moderate spectral resolution.

$\mathrm{AMBER}^{1}$ is the first-generation general-user near-infrared focal instrument of VLTI. After about two years of preliminary studies and lobbying, actual development started in 1998 after the European Southern Observatory (ESO) decided to revive the development of interferometry at the VLT (Paresce et al. 1996). The instrument was installed and obtained its first fringes in March 2004. Subsequent work has focused on technical investigations, commissioning and preliminary scientific observations. Figure 1 is a photograph of the instrument. AMBER builds on experience gained with several optical interferometers: GI2T (Mourard et al. 2003) for the principle of dispersed fringes, FLUOR (Coudé Du Foresto et al. 1998; Coudé du Foresto et al. 2003) for high accuracy with single-mode fibers, and PTI (Colavita et al. 1999; Colavita 1999) for the data reduction scheme.

Section 2 reviews the main scientific drivers of AMBER, which allowed us to define the instrumental specifications. The

\footnotetext{
1 Astronomical Multi-BEam combineR.
}

instrument concept is described in Sect. 3, together with its different parts. Section 4 discusses the expected performances of AMBER in relation with what has been measured currently. Section 5 presents the various operating modes of AMBER and discusses the calibration procedures.

\section{Science drivers and specifications}

The specifications of AMBER have been defined as giving the highest priority to three key astrophysical programs: young stellar objects (YSOs), active galactic nuclei (AGN), and hot giant extra-solar planets (ESPs). The first one was considered as the minimum objective and the third one as an ambitious goal at the very edge of what could be achieved with the technology and VLTI infrastructure expected when AMBER was to be installed. We used the experience gained with IOTA, PTI, GI2T, and single-aperture speckle interferometry on highangular-resolution instrumentation to define a certain number of strategic choices:

- operation in the near-infrared domain [1-2.5 $\mu \mathrm{m}]$;

- spectrally dispersed observations;

- spatial filtering for high-accuracy absolute visibility;

- very high-accuracy differential visibility and phase;

- imaging information from closure phase.

Table 1 lists the intersection between these strategic choices (columns) and the needs set by the scientific objectives (lines), as summarized below. Framed specifications cooresponds to the most demanding ones.

\subsection{Young stellar objects}

The study of YSOs is critical to understanding stellar and planetary formation. Seeing-limited spectrophotometry that observes down to about $100 \mathrm{AU}$ and, more recently, millimeter wave interferometry, bispectrum speckle interferometry, and adaptive 
Table 1. Summary of the initial scientific requirements and top level specifications for AMBER.

\begin{tabular}{|c|c|c|c|c|c|}
\hline Scientific topic & $\begin{array}{l}\text { Spectral } \\
\text { coverage }\end{array}$ & $\begin{array}{c}\text { Spectral } \\
\text { resolution }{ }^{a}\end{array}$ & $\begin{array}{c}\text { Minimum } K \\
\text { band magnitude }\end{array}$ & $\begin{array}{c}\text { Maximum } \\
\text { visibility error }^{b}\end{array}$ & $\begin{array}{c}\text { Imaging } \\
\text { (closure phase) }\end{array}$ \\
\hline Key programs & & & & & \\
\hline Young stellar objects & $J, H, K$, lines & medium & 9 & $10^{-2}$ & yes \\
\hline AGN dust tori & $K$ & low & 11 & $10^{-2}$ & yes \\
\hline Extrasolar planets & $J+H+K$ & low & 5 & $10^{-4}$ & no $^{c}$ \\
\hline \multicolumn{6}{|l|}{ General programs } \\
\hline Stellar structure & lines & high & 2 & $10^{-4}$ & yes \\
\hline Circumstellar envelopes & $J, H, K$ & medium & 4 & $10^{-2}$ & yes \\
\hline Binary & K & low & 9 & $10^{-3}$ & yes \\
\hline QSO and AGN BLR & $J, H, K$, lines & medium & 11 & $10^{-2}$ & no \\
\hline
\end{tabular}

${ }^{a}$ At the time when it was specified, low spectral resolution meant about 35, medium resolution about 1000 and high resolution at least 10000. ${ }^{b}$ Error on either visibility amplitude (in normalized visibility units) or differential phase (in radians). ${ }^{c}$ As phase is likely to be more critical for ESP than simultaneous $J+H+K$ observations.

optics, typically observing down to $10 \mathrm{AU}$, gave the outlines of YSO physics, but many detailed mechanism issues remain open. Long-baseline optical interferometry, with its milliarcsecond (mas) resolution typically corresponding to $0.1 \mathrm{AU}$ at 100 $\mathrm{pc}$, is therefore essential for unravelling the physics of the early stages of star and planet formation (see reviews by Malbet 2003; Millan-Gabet et al. 2007).

The small-aperture optical interferometers can access the limited number of YSO brighter than $K=7$. Since the VLTI should easily reach $K \geq 9$, AMBER should give access to hundreds of YSO candidates and allow a major breakthrough in the field. Simulations of the signatures of various wind and disk geometries have demonstrated that the accuracy needed for absolute visibility is about 0.01 . In addition to many broad band programs, moderate spectral resolution observations within emission lines like Bracket $\gamma$ at $2.1655 \mu \mathrm{m}$ would, for example, allow astronomers to characterize the stellar wind-launching regions, and to find out if it comes from the star or from the disk. Given the complexity of the immediate surroundings of young stars, even rough imaging would be welcome.

\subsection{Active galactic nuclei}

Observing galactic nuclei allows study of the physics in extreme conditions at the limits of our physical world set by the horizon of the massive black hole that they might be hosting. Accretion on such a black hole is proposed to explain the extreme energetic phenomena observed in AGN. The nearest AGN are important candidates for testing the unification schemes (Antonucci \& Miller 1985) between the two main observational categories of AGN. The main unification feature is a geometrically and optically thick torus able to obscure the central continuum source if the angle between the torus axis and the observer's viewing direction is large enough. It is believed that type 1 AGN are seen almost pole-on, whereas type 2 AGN are observed almost edgeon.

The specific requirement for observing AGN is instrument sensitivity. A coherent magnitude $K \simeq 10$ will allow the very brightest candidates to be observed. With $K \simeq 11$, several tens of AGN are within reach, so that this is the limiting magnitude specification for AMBER. AGN visibility observation would allow for constraining dust torus models, but a full demonstration of the unified AGN model would require model-independent detection of the torus, which is an image in the near infrared permitted by the AMBER closure phase.

Medium spectral-resolution observations in emission lines yields the structure of the base of the jet in the NLR. Measuring the differential phase through the line wings yields the size and kinematics of the BLR, and thereby confirming the presence of a black hole and evaluating its mass (Marconi et al. 2003). Combining this angular size with the linear one given by reverberation mapping gives direct distance measurements on a new scale. This requires a fringe tracker able to reach the same $K \simeq 11$ limit.

\subsection{Extrasolar planets}

The search for and study of extrasolar planets (ESP) is currently one of the major goals in astronomy, because it is a key ingredient in a cultural investigation of the place of life, conscience, and mankind in the Universe. We are living in a fascinating period when these philosophical questions start being answered by actual physical measurements. The surprisingly large number of giant hot planets orbiting very close to their star raises new astrophysical problems but also opens new observational opportunities. A key issue is understanding the migration mechanism, its regulation, and consequences. In particular, are hot giant planets compatible with planets at more classical locations?

Measuring the variation in the differential phase and visibility with wavelength at different orbital phases yields the planet orbit and therefore mass, the planet/star flux ratio, and the planet's low-resolution (say 30 to 50) spectrum (Vannier et al. 2006). The latest yields the planet's effective temperature. Combined with the flux ratio, it allows the radius to be computed and the mass-radius relationship to be constrained. The shape of the spectrum constrains the composition of the atmosphere but also its dustiness (Barman et al. 2001).

The expected signal is very weak $\left(\simeq 10^{-4} \mathrm{rad}\right)$, but the UTs collect enough photons to theoretically permit this level of accuracy in a few hours, if the instrument is extremely stable and all atmospheric effects are filtered and calibrated extremely well. A major difficulty is the variable chromatic OPD of dry air and of water vapor above the telescopes and in the tunnels (Akeson et al. 2000). A first approach is to instantaneously fit the differential thickness of water vapor and dry air with the other parameters. This leads to the initial requirement of simultaneous $J+H+K$ band observations ${ }^{2}$. A complementary approach (Ségransan et al. 2000) is to use the closure phase permitted by AMBER with three UTs, because it cancels all OPD effects. The closure phase remains affected by non-OPD effects, such as

\footnotetext{
2 Roughly, the $J$ band is used to fit the chromatic OPD whereas the planet signal is analyzed in $H$ and $K$.
} 


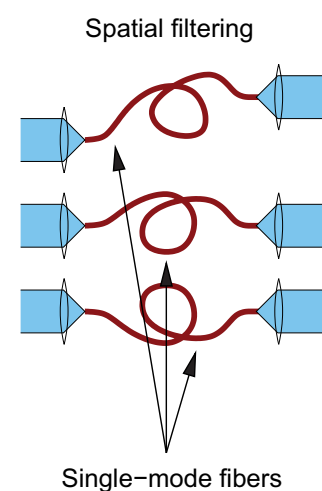

Single-mode fibers

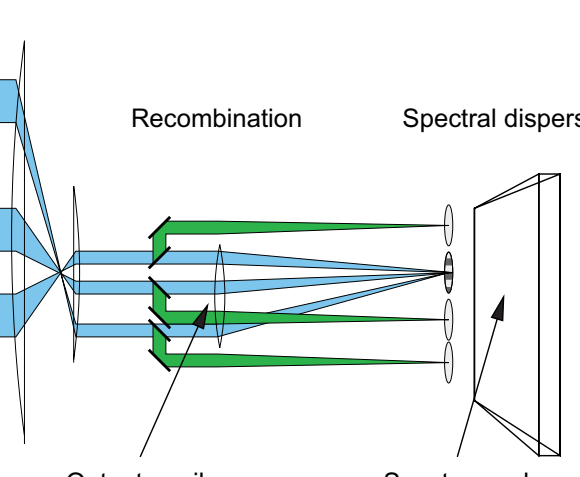

Output pupils

Spectrograph
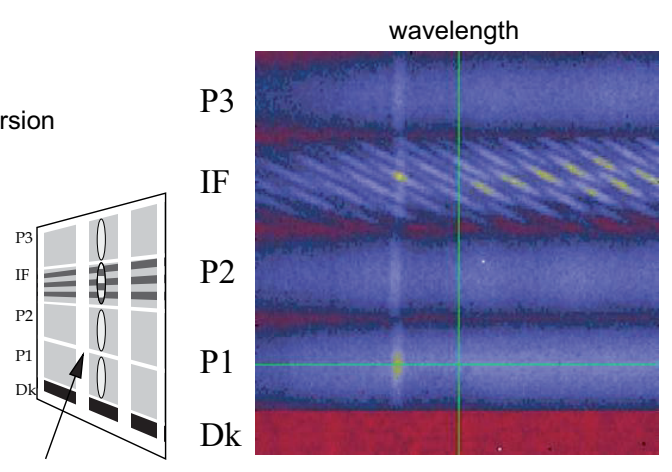

Fig. 2. The basic concept of AMBER. First, each beam is spatially filtered by a single-mode optical fiber. After each fiber, the beams are collimated so that the spacing between the output pupils is non redundant. The multiaxial recombination consists in a common optics that merges the three output beams in a common Airy disk containing Young's fringes. Thanks to a cylindrical optics anamorphoser, this fringed Airy disk is fed into the input slit of a spectrograph. In the focal plane of the spectrograph, each column (in the figure, but in the reality each line) of the detector contains a monochromatic image of the slit with 3 photometric (P1, P2, P3) zones and one interferogram (IF). In this figure, the detector image contains a view rotated of $90^{\circ}$ of the AMBER real-time display showing three telescope fringes, in medium-resolution between 2090 and $2200 \mathrm{~nm}$, on the bright Be star $\alpha$ Arae. The three superimposed fringe patterns form a clear Moiré figure because, in that particular case, the three OPDs were substantially different from zero during the recording. Note that the vertical brighter line indicating the $\mathrm{Br} \gamma$ emission line.

detector variations and data processing biases that should vary slowly enough to be eliminated by an internal modulation of the instrumental closure phase, as explained in Sect. 5.5.

\subsection{Broader program and additional requirements}

With the specifications and goals set from the three key programs, AMBER can also be used for constraining fundamental stellar parameters such as mass, radius, and age, and for stellar activity studies, such as of star spots and non radial pulsations. A broader program includes:

- Late stages of stellar evolution. The late stages of stellar evolution play a crucial role in understanding the chemical evolution of our Galaxy, since the peeling of stellar surfaces by strong stellar winds leads to the chemical enrichment of the interstellar medium. The specific needs of these programs are very high angular resolution (for example by using the AT telescopes on the longest possible baselines), and high spectral resolution (10000 or more) to be able to spectrally select active features like spots or supergranules;

- Environment of hot stars. Be or B[e] stars exhibit strong emission in the hydrogen lines and are believed to be surrounded by a large circumstellar rotating and/or an expanding envelope. Differential visibility and phase constrain the size, shape, and kinematics of the envelope and help to find the balance between envelope rotation and latitudedependent radiation pressure. The closure phases yield independent constraints on the envelope asymmetries and will eventually allow reconstructions of the images able to test differential interferometry deductions;

- Massive stars. Their complex structure is affected by dramatic events over relatively short periods of time. In the first stages of their evolution, their powerful stellar radiation ionizes their close environment in $\mathrm{H}_{\mathrm{II}}$ region and feeds the interstellar medium with high-velocity material. AMBER will resolve the close multiple systems that were not identified with observations at lower spatial resolution. The spectral resolution of AMBER will disentangle the complex kinematics of the close surroundings of luminous blue variables (LBVs) like the typical Eta Carinae system.

The high spectral-resolution mode will be accessible only on a small number of bright targets, except if an external fringe tracker allows us to stabilize the fringes and to observe with long (a few seconds) frame times with AMBER. The VLTI plans include a three telescopes fringe tracker, called FINITO ${ }^{3}$, operating in the $H$ band, and leaving all the flux in $J$ and $K$ and a fraction of the flux in $H$ for AMBER.

\section{The AMBER concept}

This section is intended to provide the reader (and hopefully the future user of AMBER) with an overview of the key conceptual characteristics of AMBER. The optical principle (Sect. 3.1) and the data reduction (Sect. 3.2) are described in much greater details in other papers in this volume (Robbe-Dubois et al. 2007; Tatulli et al. 2007b). Here, the reader should find a summary and a brief justification of the fundamental choices made in the design of AMBER and its data processing. The selection of the concept is the result of an iteration between scientific specifications, performance, and complexity estimates in the context of some preferences set by previous experience with interferometric instruments. Section 3.3 insists on the AMBER observables and on the type of source constraints that can be expected from them.

\subsection{Optical principle}

Figure 2 summarizes the key elements of the AMBER concept. It was designed following a multiaxial beam combination, namely an optical configuration similar to the Young's slits experiment, which overlaps images of the sources from different telescopes. A set of collimated and mutually parallel beams are focused by a common optical element in a common Airy pattern that contains fringes. The output baselines are in a non redundant set up, i.e. the spacing between the beams is selected for the Fourier transform of the fringe pattern to show separated fringe peaks at all wavelengths. The Airy disk needs to be sampled by many pixels in the baseline direction (an average of 4 pixels in the narrowest fringe, i.e. at least 12 pixels in the output baseline direction), while in the other direction a single pixel is sufficient. To minimize detector noise, each spectral channel is concentrated

\footnotetext{
${ }^{3}$ Initially, FINITO should have been operational well before AMBER. At the date of writing of this paper, FINITO had been successfully operated with the ATs and is about to be offered in combination with AMBER in 2007.
} 
amber image "AMBER.2006-04-16T04:28:45.824.fits" Frame $\mathrm{n}^{0} 7 / 1000$

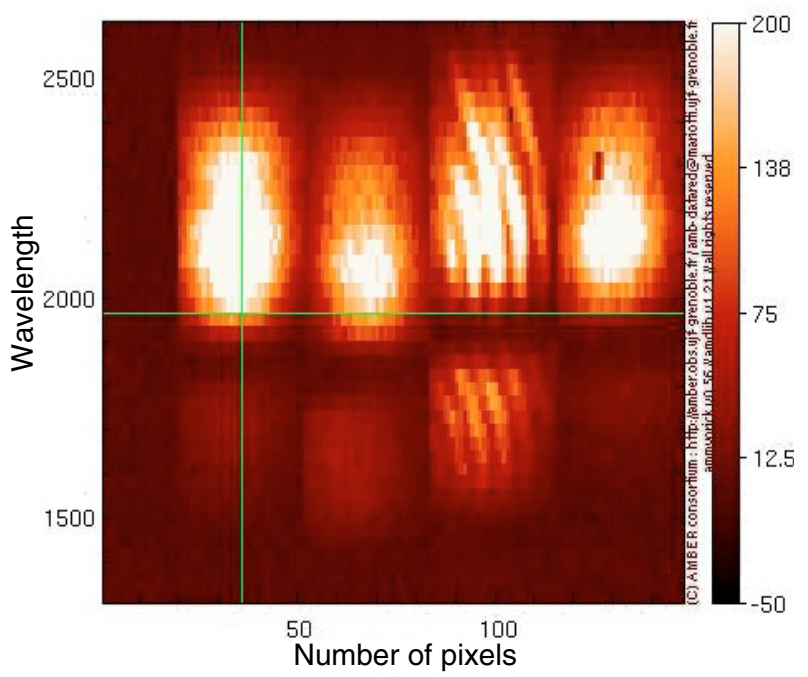

Fig. 3. Raw detector image from amber obtained in the three-telescope low-resolution mode on the star $\tau$ Bootis in April 2006. The stellar signal is spectrally dispersed in the vertical direction and each (pair of) detector line contains a spectral channel. The rows of $K$ band and $H$ band occupy respectively the upper and the lower half of the screen. From left to right, the first, second, and fourth columns represent the photometric beams for the each one of the free telescopes, while the third column contains the interferometric signal, with three superimposed fringe systems.

in a single column of pixels by cylindrical optics. This multiaxial beam combiner has been selected because it allows an easy and modular evolution from two to three, or even 4, telescopes and because it simplifies the design of the interface to the spectrograph and of the spectrograph itself. This is obtained without loss of potential SNR compared to a coaxial scheme like FLUOR (Coudé Du Foresto et al. 1998).

The fringes are dispersed by a standard long-slit spectrograph on a two-dimensional detector. To work in the $K$ band with resolutions up to 10000 , the spectrograph must be cooled down to about $-60{ }^{\circ} \mathrm{C}$ with a cold slit in the image plane and a cold pupil stop. In practice, it is simpler to cool it down to the temperature of liquid nitrogen.

To produce highly accurate measurements, it is necessary to spatially filter the incoming beam's wavefronts to force each one of them to contain only a single coherent mode. To ensure the most accurate visibility measurements, the spatial filter must transmit at least $10^{3}$ more light in the main guided mode than in all the secondary modes. For the kind of imperfect AO correction (Strehl ratios often below 50\%) available for the VLTI, the only way to achieve this filtering quality with decent light transmission is to use single-mode optical fibers. The wavefront corrugations are translated in time-dependent intensity fluctuations and a global chromatic "piston". The word "piston" designates the average of the wavefront OPD on each telescope aperture. The flux transmitted by each fiber must be monitored in real time for each spectral channel. A fraction of each beam is extracted before the beam combiner and sent directly to the detector through a dispersive element. The instrument must also perform some other individual beam processing before entering the spatial filter, such as correcting for the differential atmospheric refraction in the $H$ and $J$ bands or, in some cases, eliminating one polarization.

Figure 3 displays the raw image that is recorded by the amber detector (see caption for details) in low-resolution mode where the spectrum from 1.4 to $2.5 \mu \mathrm{m}$ is present. In medium and high resolution mode only a part of one NIR band is recorded.

The description above applies to one spatial filter. Since single-mode fibers are not fully efficient from 1 to 2.5 microns and the sampling on the detector depends linearly with the wavelength, AMBER has been designed to have 3 working spatial filters in $J, H$, and $K$. The parameters have been optimized for each band: numerical aperture of the single mode fiber, size of the output pupils, and separation between the different apertures. Dichroics separate the incoming beams in 3 beams, which are processed separately (from left for $K$ to right for $J$ in Fig. 2). Output dichroics merges the beams again at the output of the fibers. The global sizes and spacings of the output pupils in $J$, $H$, and $K$ are set proportionally to the central wavelength of each filter, so that the Airy disk and inter fringe at 1.25, 1.65, and $2.15 \mu \mathrm{m}$ are the same on the detector.

An important additional device, which is not represented in Fig. 2, is the calibration and alignment unit (CAU) used to feed AMBER with the well-characterized artificial fringes needed for the interferometric P2VM calibration described below.

A polarization maintaining single-mode fiber introduces a random OPD between the two polarizations. In the absence of a compensator, this effect can destroy the instrumental contrast. To avoid this, AMBER rejects one of the polarizations of the incoming beams. This results in the loss of $50 \%$ of the photons. It is important to note that this polarization direction is fixed according to the interferometric table and is therefore variable when projected on the sky. When model-fitting polarized objects, one should take this effect into account.

The subsystems of AMBER, their specifications, designs, and tests are described in detail in Robbe-Dubois et al. (2007).

\subsection{Basic AMBER measures}

\subsubsection{Access to the $u-v$ plane}

The VLTI gives access to the complex visibility function of the source at a limited number of spatial frequencies $\vec{B}^{l m} / \lambda$, where $\vec{B}^{l m}$ is the baseline that joins telescopes $l$ and $m$ projected on the sky. If $o_{*}(\vec{\alpha}, \lambda)$ is the source brightness distribution at the angular position $\vec{\alpha}$ and the wavelength $\lambda$, the source complex visibility function is defined by the Fourier transform:

$O_{*}(\vec{u}, \lambda)=\int o_{*}(\vec{\alpha}, \lambda) \mathrm{e}^{-2 \mathrm{i} \pi \vec{\alpha} \vec{u}} \mathrm{~d} \vec{\alpha}$

At the spatial frequency $\vec{u}=\vec{B}^{l m} / \lambda$, this is

$O_{*}\left(\vec{B}^{l m} / \lambda, \lambda\right)=O_{*}^{l m}(\lambda)=V_{*}^{l m}(\lambda) \mathrm{e}^{\mathrm{i} \phi_{*}^{l m}(\lambda)}$

where $V_{*}^{l m}(\lambda)$ and $\phi_{*}^{l m}(\lambda)$ are the amplitude and phase of the complex visibility $O_{*}\left(\vec{B}^{l m} / \lambda, \lambda\right)$. When the word "visibility" is used alone, it refers to the amplitude $V_{*}^{l m}(\lambda)$.

The wavelength dependence of the spatial frequency transforms the AMBER large spectral coverage from 1.05 to $2.4 \mu \mathrm{m}$ in an important increase in spatial coverage as illustrated by Fig. 4, which has to be considered when preparing AMBER observations.

\subsubsection{Data set in AMBER frames and the interferometric equation}

For each elementary frame and in each AMBER spectral channel, i.e. in each line of the detector, we have an interferometric and three photometric signals that have been processed by 


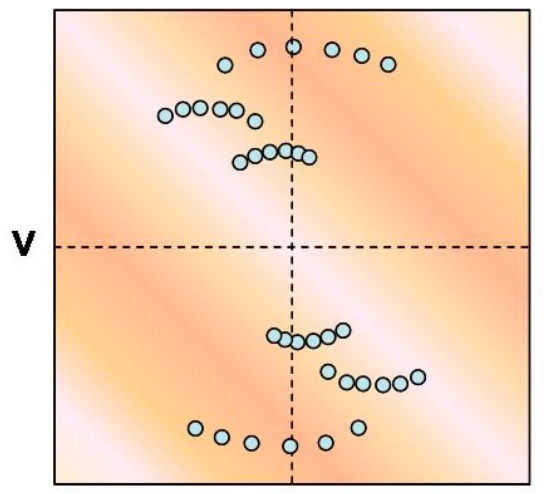

$\mathbf{U}$

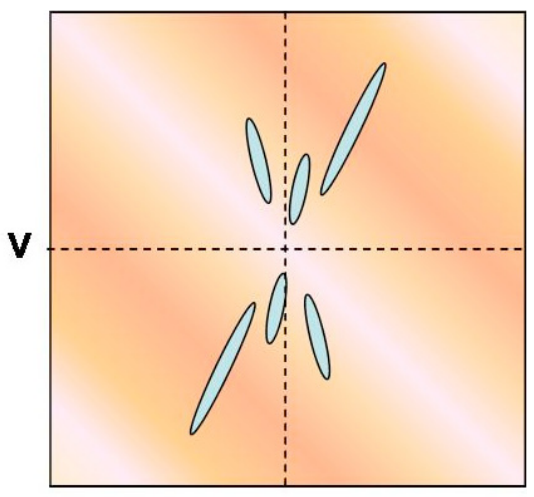

$\mathbf{u}$
Fig. 4. Exploration of the u-v plane using spectral coverage. The two figures represent the typical fringed visibility function as a function of $\mathrm{u}$ and $\mathrm{v}$, as produced by a binary. On the left we have the u-v tracks obtained with a single spectral channel and 10 hours of observations with UT1-UT3-UT4. On the right, we represent the $\mathrm{u}-\mathrm{v}$ coverage obtained from 1.05 to 2.4 microns with only one snapshot observation with the same telescopes. As demonstrated by (Millour et al. 2007), the constraints on the binary angular separation are similar for the left and right $\mathrm{u}-$ $\mathrm{v}$ coverages, but in the spectrally resolved case we obtain the spectra of the components in addition. the same optics and the same dispersive elements (as shown in Figs. 2 and 3). For a single-mode instrument, the Fourier interferogram (Fourier transform of the fringe pattern in the interferometric channel) is

$$
\begin{aligned}
& I(\vec{u}, \lambda)=P(\vec{u}, \lambda) \sum_{1}^{n_{T}} n_{*}^{i}(\lambda) \\
& +P\left(\vec{u}-\frac{\vec{B}^{i j}}{\lambda}, \lambda\right) \sum_{i \neq j} \sqrt{n_{*}^{i}(\lambda) n_{*}^{j}(\lambda)} V_{m}^{i j}(\lambda) \mathrm{e}^{\mathrm{i} \phi_{m}^{i j}(\lambda)}
\end{aligned}
$$

where $P(\vec{u}, \lambda)$ is the single-telescope transfer function at the spatial frequency $\vec{u}$ and wavelength $\lambda, V_{m}^{i j}(\lambda)$ and $\phi_{m}^{i j}(\lambda)$ are the measured visibility and phase and $n_{*}^{i}(\lambda)$ is the total contribution of beam $i$ to the source flux collected in the interferometric channel. In the following we note $n_{*}^{i}, V_{m}^{i j}$ and $\phi_{m}^{i j}$ for $n_{*}^{i}(\lambda), V_{m}^{i j}(\lambda)$ and $\phi_{m}^{i j}(\lambda)$ respectively, except when the wavelength dependence is explicitly used.

\subsubsection{Basic Fourier estimator}

The first term in Eq. (3) is the low-frequency peak affected only by a fixed instrumental term $P(\vec{u})$ and scaled by the total number of photons $\Sigma n_{*}^{i}$ collected in all beams. The second term is the fringe peak, with a phase $\phi_{m}^{i j}$ and an amplitude equal to the measured coherence flux $\sqrt{n_{*}^{i} n_{*}^{j}} V_{m}^{i j}$ at frequency $\vec{B}^{i j} / \lambda$. The number of photons $n_{*}^{i}$ detected in each frame are deduced from the photometric channels. In an ideal interferogram where the fringe peaks are fully separated, the measured visibility is obtained by dividing the Fourier interferogram at frequency $\vec{u}=\overrightarrow{B^{i j}} / \lambda$ by its value at frequency $\overrightarrow{0}$ and correcting by the known photometry:

$V_{m}^{i j} \mathrm{e}^{\mathrm{i} \phi_{m}^{i j}}=\frac{I\left(\vec{B}^{i j} / \lambda\right)}{I(0)} \frac{\sum n_{*}^{i}}{\sqrt{n_{*}^{i} n_{*}^{j}}} \mathrm{e}^{-\mathrm{i} \phi_{P}^{i j}}$

The $\mathrm{e}^{-\mathrm{i} \phi_{P}^{i j}}$ corresponds to a correction of the effect of the differential piston between beams $i$ and $j$. The measured visibility is

$V_{m}^{i j}(\lambda)=V_{*}^{i j}(\lambda) C_{I}^{i j}(\lambda)$

where $C_{I}^{i j}(\lambda)$ contains internal AMBER instrumental terms $C_{A}^{i j}(\lambda)$, which are removed by the calibration procedure below. It is also affected by the "piston jitter", i.e. the variation of the piston during acquisition of the frame. If $\Delta p^{i j}$ is this jitter, supposed to be smaller than the total piston excursion (because we use short frame times), we can consider that fringes were drifting almost linearly during the frame and the contrast loss is then given by

$C_{I}^{i j}(\lambda)=C_{A}^{i j}(\lambda) \frac{\sin \left(\pi \Delta p^{i j} / \lambda\right)}{\pi \Delta p^{i j} / \lambda}$,

which can be corrected if the piston jitter within one frame can be deduced from the piston variations between two consecutive frames.

\subsubsection{Modal visibility of a single-mode instrument}

A single-mode fiber collects light within an "antenna" lobe $f(\vec{\alpha})$ describing the relative contribution of a point on the sky at an angular distance $\vec{\alpha}$ of the pointing direction. The source observed by AMBER is integrated and weighted by this "antenna lobe" The instantaneous antenna function depends on the geometry of the fiber, and does so for each aperture PSF partially corrected by adaptive optics. The exact measured visibility also depends on the degree of coherence between the antenna functions of each individual aperture. Single-mode interferometers give access only to the "modal" visibility defined by relatively complex relations to be found in Mège et al. (2003). It is important to note that:

- For sources smaller than the Airy disk of individual apertures, the modal visibility is equal to the source visibility;

- For extended sources, there is no simple way to separate the contributions of the source and of the individual aperture PSF. What has been done so far (van Boekel et al. 2003) is to consider the object as the sum of a small source, for which the above applies, and of a source substantially larger than the Airy disk, which produces a completely incoherent contribution. When this is wrong, one should use the modal visibility in the model-fitting procedure, which assumes a model of the average adaptive-optics corrected PSF. Some simplifications can be found for particular objects, such as double stars.

A full discussion of this topic is beyond the scope of this paper. To our last present knowledge, the problem has not yet been resolved for a general source. We now say that AMBER (and other VLTI or KeckI single-mode instruments) perform well when the source is smaller than the individual aperture Airy disks (which is a severe restriction for interferometers with small baseline/diameters ratios). In the following we do not distinguish 
between the modal and the true complex visibility so use $V_{*}^{i j} \mathrm{e}^{\mathrm{i} \phi_{*}^{i j}}$ for both.

\subsubsection{The pixel to visibility approach}

Fully separating the fringe peaks in a multi-axial beam combiner implies a large separation between output pupils (i.e. many fringes per Airy disk) and a window that is much larger than the Airy disk and would thus imply reading a very large number of pixels, which is not acceptable when one is limited by detector read-out noise. To overcome the problem of partially overlapping fringe peaks, a generalization of the ABCD algorithm used in the PTI interferometer (Colavita 1999) has been developed to establish a linear relation between the values measured in each pixel and the complex coherence for each baseline (for details see Tatulli et al. 2007b). Fundamentally, the technique consists in finding the modulus and phase for each baseline, which allows the calibrated fringe shapes (the carrying waves) to be fitted in the data. This fit is linear and is based on the visibility-to-pixel matrix, computed from a set of calibration measurements using the artificial coherent source unit (CAU) of the instrument. These measurements are the photometry and interferometry from each beam alone and the interferograms for each pair of beams at 0 and close to $\lambda / 2$ OPD. This matrix is then inverted to get the pixel to visibility matrix (P2VM) used to obtain the complex visibility in each frame from the intensities measured in each pixel. This P2VM matrix takes all stable instrumental effects into account, such as the detector gain table, the shape and the overlap of the output fiber beams, and the instrument's chromatic OPD.

Analytical computations and numerical simulations show that this P2VM approach can be implemented without any SNR cost (Tatulli \& LeBouquin 2006), at least when the SNR per frame is larger than 1 .

\subsection{AMBER observables}

During the observation, we record NEXP exposures of NDIT frames, each exposed during the detector integration time, DIT. Typically a calibrated point is made of NEXP $=5$ exposures of NDIT $=1000$ frames of DIT $=20$ to $100 \mathrm{~ms}$. For each baseline $l m$, and in each spectral channel, we obtain a measure of the intensity and of the visibility amplitude $V_{m}^{l m}(\lambda)$ and phase $\phi_{m}^{l m}(\lambda)$, used to derive the various AMBER observables.

\subsubsection{Spectrum of the source}

Each one of the photometric beams yields a spectrum of the source within the chosen spectral window. The raw AMBER spectrum must be calibrated in intensity and in wavelength, which in medium and high resolution modes requires acquiring a spectral calibrator with a known continuum shape and spectral features as illustrated by Fig. 5. The spectrum is a crucial element of AMBER model fitting. Simultaneous observations of high-resolution infrared spectra, for example with the ISAAC instrument, have often been found to be very useful (Meilland et al. 2007b; Malbet et al. 2007).

\subsubsection{Absolute visibility per spectral channel}

The absolute visibility in each spectral channel is the direct result of the P2VM data processing. As discussed above, the measured absolute visibility is affected by the piston jitter within one frame. This jitter should be frozen by VLTI fringe tracking or measured when the flux per frame is high enough. Currently this correction is impossible because the vibrations in the Unit

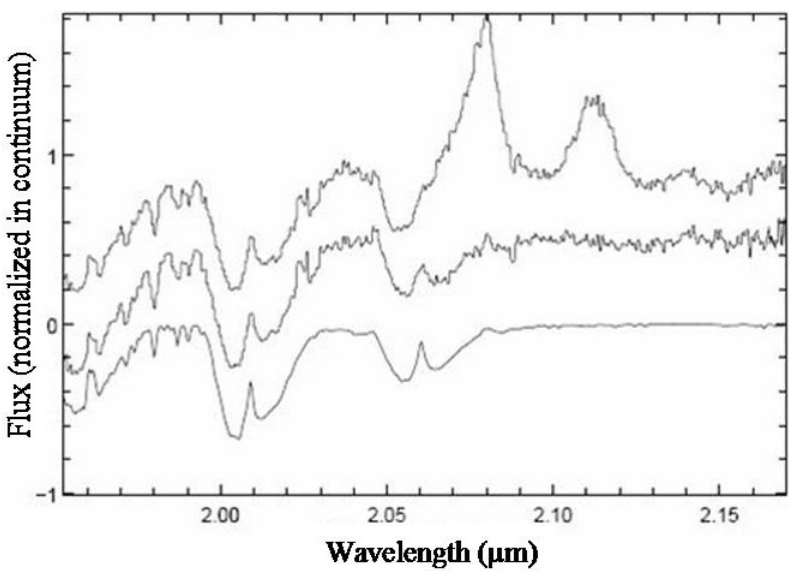

Fig. 5. Data used for spectral calibration. Top: source spectrum $\left(\gamma^{2} V e l\right)$; Middle: the calibrator star flat spectrum (shifted by -0.5 ); Bottom: the reference Gemini spectrum (shifted by -1 ). Note the atmospheric watervapor doublets at 2.01 and $2.06 \mu \mathrm{m}$ used for calibration in $K$-band medium-resolution observations.

Telescope produce a piston variation that is almost always larger than $\lambda$ between consecutive frames. A partial correction of the effect is obtained using a calibrator star:

$V_{\mathrm{s}}(\lambda)=\frac{V_{\mathrm{ms}}(\lambda)}{V_{\mathrm{mc}}(\lambda)} V_{\mathrm{c}}(\lambda)$,

where $V_{\mathrm{s}}(\lambda)$ is the calibrated visibility of the target, $V_{\mathrm{ms}}(\lambda)$ and $V_{\mathrm{mc}}(\lambda)$ are the visibilities measured on the target and on the calibrator, and $V_{\mathrm{c}}(\lambda)$ is the known visibility of the calibrator. This correction is poor ( 0.03 to 0.07 visibility accuracy), if we cannot apply a frame-by-frame piston jitter correction. Then, it is particularly important to have a frame-selection criterion, independent of flux and tending to select an equivalent set of frames for the science and the calibration source. Currently the best solution is to select calibrators with magnitudes comparable to the science source and to select the same percentage of best contrasts in both cases. Even with an excellent jitter correction, using a calibrator will always be necessary for good accuracy measurements to compensate for slow instrumental variations modifying the value of all the second-order instrumental defects. For example, a small change in the position of the CAU beams will change the contrast of the CAU source and therefore the corrected instrumental contrast.

The absolute visibility mainly depends on the equivalent size of the source in the direction of the baseline. A fit of absolute visibility as a function of baseline length allows us to estimate the equivalent radial intensity profile, such as the limb darkening. This has been illustrated by AMBER observations of $\eta$ Carinae that permitted Weigelt et al. (2007) to obtain a remarkable fit of the central-wind limb-darkening by Hillier \& Lanz (2001) model. A fit of visibility as a function of position angle of the projected baseline yields the source anisotropy, which can be related to flattened inclined disks (Malbet et al. 2007), sometimes combined with a very strong polar flow (Meilland et al. 2007b) or an optical wind enhanced in the polar direction of a fast rotator (Domiciano de Souza et al. 2005; Weigelt et al. 2007) or a combination of all these factors. Distinguishing between these possibilities requires prior knowledge of the source. Visibility alone does not allow axisymmetric and non axisymmetric solutions to be disentangled between (except with a very good $u-v$ coverage) and will be of little use if the structure of the objet is completely unknown. 


\subsubsection{Differential visibility}

The differential (or relative) visibility $V_{\mathrm{ds}}(\lambda)$ is the source visibility in the spectral channel $\lambda$, often called work channel, calibrated by the average visibility of a reference channel:

$V_{\mathrm{ds}}(\lambda)=\frac{V_{\mathrm{ms}}(\lambda)}{V_{\mathrm{ref}}(\lambda)}$

where

$V_{\mathrm{ref}}\left(\lambda_{j}\right)=\frac{1}{n_{\lambda}-2}\left(\sum_{i=1}^{n_{\lambda}} V_{\mathrm{ms}}\left(\lambda_{i}\right)-V_{\mathrm{ms}}\left(\lambda_{j}\right)\right)$.

This is the standard way to build the reference channel in AMBER data processing. The users can choose many other definitions of the reference channel, such as using selected continuum channels. The ideal is, of course, to use channels where the source is known or particularly well-constrained, but in any case, the exact definition of the reference channel, which depends of the source and of data processing constraints, must be injected in the model fitting. The important feature is that the differential visibility is independent of most of the systematic effects affecting the absolute visibility, and it does not need the use of a calibrator star. In particular, the differential visibility will be insensitive to the piston jitter over a limited wavelength range.

The relative visibility basically yields the same physical information as the spectrally-resolved absolute visibility, but it is much better calibrated, at the cost of losing information on the reference channel.

\subsubsection{Differential phase}

In optical, as well as in radio astronomy, source phase information refers to the phase of its complex visibility. In a single-mode interferogram, the phase is related to the position of the fringes, and in the absence of nanometer accuracy metrology, the measured phase $\phi_{\mathrm{ms}}(\lambda)$ is affected by an unknown instrumental term linked to the VLTI+AMBER differential piston $\delta_{l m}$ and to the instantaneous atmospheric piston $p_{l m}$ between beams $l$ and $m$ :

$\phi_{\mathrm{ms}}(\lambda)=\phi_{\mathrm{s}}(\lambda)+2 \pi(\delta+p) / \lambda$.

Introducing a development of the source phase $\phi_{\mathrm{s}}(\lambda)$, as a function of the wave number $\sigma=1 / \lambda$ :

$\phi_{\mathrm{s}}(\lambda)=a_{0}+a_{1} \sigma+\varphi_{\mathrm{sh}}(\lambda)=\varphi_{\mathrm{sl}}(\lambda)+\varphi_{\mathrm{sl}}(\lambda)$

where $\varphi_{\mathrm{sl}}(\lambda)$ stands for the straight line that can be fitted through the measured phase and that contains the piston residual, as well as the equivalent piston of the source phase averaged over the reference channel. The higher terms of the phase polynomial development $\varphi_{\mathrm{sl}}(\lambda)$ are the source information, which can be extracted from differential phase measurements. We first fit the measured phase $\phi_{\mathrm{ms}}(\lambda)$ phase with a linear function of $\sigma$. Then we correct the coherent flux $C(\lambda)$ for this linear phase phasor, and we integrate it in a reference channel:

$C_{\text {ref }}\left(\lambda_{j}\right)=\sum_{i=1}^{n_{\lambda}} C\left(\lambda_{i}\right) \mathrm{e}^{-\mathrm{i} \varphi_{s l}\left(\lambda_{\mathrm{i}}\right)}-C\left(\lambda_{j}\right) \mathrm{e}^{-\mathrm{i} \varphi_{\text {sl }}\left(\lambda_{j}\right)}$.

The differential phase is defined by:

$\varphi_{\mathrm{ds}}(\lambda)=\arg \left(\left\langle\left(C(\lambda) C_{\mathrm{ref}}^{*}(\lambda)\right\rangle\right)\right.$.
If the reference channel has been defined in channels where the source phase is equal to 0 , for example in the spectral continuum channel of a point-like or a symmetrical source,

$C_{\text {ref }}(\lambda)=\Sigma_{\lambda_{\text {cont }}} C(\lambda) \mathrm{e}^{-\mathrm{i} \varphi_{\mathrm{sl}}(\lambda)}=V_{\mathrm{ref}} \mathrm{e}^{-2 \mathrm{i} \pi(\delta+p) / \lambda}$

This yields the higher-order term $\varphi_{\mathrm{sl}}(\lambda)$. We obtain phase information on the source, but we have lost the equivalent piston of the reference channel.

As for the differential visibility, there are many ways to define the reference channel, which must be injected in the source model-fitting process. Equation (14) gives the definition of the reference channel used in AMBER data processing.

A remarkable feature of the differential phase is that, for non resolved (i.e. smaller than $\lambda / B$ ) sources, it is proportional to the variation of the photocenter of the source (Petrov 1989). Given a sufficient SNR, the photocenter variation with $\lambda$ can be measured on very unresolved sources with many very rich astrophysical applications. This includes unresolved circumstellar disks and imaging of unresolved spotted stars (Petrov 1988) or of non radial oscillation modes (Jankov et al. 2002). One of the most promising applications is the resolution and the kinematics of QSO BLR. With a fringe tracking up to $K \simeq 12$, one should be able to measure photocenter variations and therefore direct distances for some Cepheids of the Magellan Cloud.

\subsubsection{Closure phase}

The closure phase between baselines $\overrightarrow{B_{12}}, \overrightarrow{B_{23}}$ and $\overrightarrow{B_{31}}$ is the phase of the average "bispectral product" of the coherent fluxes

$\psi_{123}=\arg \left(\left\langle C_{12} C_{23} C_{31}^{*}\right\rangle\right)$,

and it is a very classical property of long-baseline interferometry that this closure phase is independent of any OPD terms affecting individually each beam. This includes the achromatic piston, as well as the chromatic OPD:

$\psi_{123}=\phi_{* 12}+\phi_{* 23}-\phi_{* 13}+\phi_{d 12}+\phi_{d 23}-\phi_{d 13}$

where $\phi_{d i j}$ are error terms linked to the baseline $i j$, due for example to a change in the detector gain table since the calibration of the P2VM. Usually, these terms are very small so it can be considered that for many applications the closure phase is only a function of the source. Unlike the differential phase, the closure phase is independent of any assumption on a reference channel. A sufficient number of closure phases, combined with accurate visibility measurements, allows reconstruction of relatively complex images, as demonstrated by the 2006 "image reconstruction beauty contest" (Lawson et al. 2006), which was based on "realistic", low-contrast, AMBER 3AT data, obtained over three simulated nights of observations. The best three image reconstructions were quite good, demonstrating a real imaging capability with a three-telescope optical interferometer.

For any triplet of baselines, the closure phase is zero for any axisymmetric object. For non-axisymmetric candidates, the closure phase decreases with the third power of the object angular size when it is getting unresolved. Then, a non zero closure phase is a strong indication of a source with an interferometrically resolved non-axisymmetric feature, like the binary system $\gamma^{2}$ Velorum (Millour et al. 2007) or the $\eta$ Carinae wind in the blue part of the Br $\gamma$ line (Weigelt et al. 2007). 


\section{AMBER current and potential performances}

In this section we first present the expected performances of AMBER on the VLTI and then briefly discuss their current status with some indications of the cause and the predictable evolution of limitations.

\subsection{Fundamental error on the visibility}

The AMBER measures are affected first by the photon noise of the source and of the background and by the detector read-out noise, called the fundamental noises. In the following we give the errors on the visibility and the phases using the standard Fourier formalism (among many others, see for example Petrov et al. 1986), which has been shown to be sufficient for SNR estimations during AMBER commissioning, when we compared Fourier SNR estimation and statistics on the measures and found them in good agreement well within a factor $\sqrt{2}$. From the interferometric Eq. (3) and the complex visibility estimator 4 it is possible to derive the error on the visibility estimator (with the approximation that all noises and pixels are statistically independent):

$$
\begin{aligned}
\sigma_{V_{i j}}= & \frac{1}{\sqrt{n_{i} n_{j}}}\left[\sqrt{\sum_{i} n_{* i}+\sum_{i} n_{\mathrm{B} i}+n_{\mathrm{p}} \sigma_{\mathrm{R}}^{2}}\right. \\
& \left.+\frac{V_{m}^{i j}}{2}\left(\frac{\sigma_{* i}}{2 \sqrt{n_{* i}}}+\frac{\sigma_{* j}}{2 \sqrt{n_{* j}}}\right)\right]
\end{aligned}
$$

where $n_{* i}$ and $n_{\mathrm{B} i}$ are the number of source and background photons per frame in one spectral channel, $n_{\mathrm{p}}$ is the number of pixels used to record one spectral channel, and $\sigma_{\mathrm{R}}$ is the detector read out noise per pixel. The first term of this equation results from the contribution of fundamental noises to the error on the coherent flux and the second term results from the photometric correction applied to obtain the visibility from the coherent flux (Tatulli et al. 2007b). The value of $\sigma_{* i}$ is the relative error on the measure of the contribution of beam $i$ to the flux in the interferometric channel:

$\sigma_{* i}=\frac{\sqrt{n_{* i}+n_{\mathrm{B} i}+n_{\mathrm{p}} \sigma_{\mathrm{R}}^{2}}}{n_{* i}}$.

In AMBER, the contribution of the thermal background is smaller than the read-out noise for all the usual short exposure times $(\leq 100 \mathrm{~ms})$ except after $2.3 \mu \mathrm{m}$. Then two cases must be considered. At high flux, when $n_{* i}>n_{\mathrm{p}} \sigma_{\mathrm{R}}^{2}$, the relative error on the visibility is given by

$\frac{V_{m}^{i j}}{\sigma_{V_{i j}}}=\frac{V_{m}^{i j} \sqrt{M n_{*}}}{\sqrt{n_{\mathrm{T}}}}$

where $M$ is the number of frames and $n_{*}$ is the average number of photons per channel, per frame, and per telescope, which are supposed equal for all telescopes. The performance of AMBER, defined here as, for example, the time needed to achieve a given SNR, is proportional to $n_{*} V^{2}$. Then, the coherent magnitude is given by the flux $n_{*} V^{2}$. This applies for relatively bright and lowcontrast sources. At lower flux, when $n_{* i}<n_{\mathrm{p}} \sigma_{\mathrm{R}}^{2}$,

$$
\frac{V_{m}^{i j}}{\sigma_{V_{i j}}}=\frac{n_{*} V_{m}^{i j}}{\sqrt{n_{\mathrm{p}} \sigma_{\mathrm{R}}^{2}}\left(1+V_{m}^{i j} / 2 \sqrt{n_{*}}\right)} \simeq \frac{n_{*} V_{m}^{i j}}{\sqrt{n_{\mathrm{p}} \sigma_{\mathrm{R}}^{2}}} \text {. }
$$

The performance of AMBER is proportional to $\left(n_{*} V\right)^{2}$ and the coherent magnitude is given by the flux $n_{*} V$. This case represents faint sources with whatever contrasts.
Table 2. Number of photons per frame and per channel needed for the frame to contribute to the ensemble averages, as a function of the observing mode and considering the measured and the potential (vibration-free) atmosphere+VLTI+AMBER visibility.

\begin{tabular}{lcc}
\hline \hline Mode & actual Visibility & potential Visibility \\
\hline Low & $V_{\text {actual }}=0.12$ & $V_{\text {potential }}=0.5$ \\
Resolution & 581 phots & 187 phots \\
\hline Medium & $V_{\text {actual }}=0.14$ & $V_{\text {potential }}=0.6$ \\
Resolution & 507 phots & 166 phots \\
\hline
\end{tabular}

Table 3. Number of photons collected per $50 \mathrm{~ms}$ frame and per spectral channel in the $K$ band in different observation modes.

\begin{tabular}{lcc}
\hline \hline Observation & best $20 \%$ & average \\
\hline Medium Resolution & 1000 & 530 \\
$K=4.9$ & & \\
\hline Low Resolution & 4250 & 2540 \\
$K=8.2$ & & \\
\hline
\end{tabular}

Table 4. Current, potential, and achieved limiting magnitudes of AMBER in the $K$ band, for $50 \mathrm{~ms}$ frames.

\begin{tabular}{lcccc}
\hline \hline $\begin{array}{l}\text { Mode } \\
\text { Conditions }\end{array}$ & $\begin{array}{c}\text { MR } \\
\text { best 20\% }\end{array}$ & $\begin{array}{c}\text { MR } \\
\text { average }\end{array}$ & $\begin{array}{c}\text { LR } \\
\text { best 20\% }\end{array}$ & $\begin{array}{c}\text { LR } \\
\text { average }\end{array}$ \\
\hline Current & 5.6 & 4.9 & 10.2 & 9.7 \\
Potential & 6.8 & 6.1 & 11.5 & 10.9 \\
\hline Achieved & - & 4.9 & - & 8.6 \\
\hline
\end{tabular}

\subsection{Limiting magnitude}

The limiting magnitude of AMBER is set by $\frac{V_{m}^{i j}}{\sigma_{V}} \simeq 1$ for the average spectral channel. The actual data processing shows that frames not complying with this criteria yield absurd estimated complex visibility that destroy ensemble averages. In practice, such frames are eliminated in the data processing. During the first commissioning of AMBER, frame times between $50 \mathrm{~ms}$ and $100 \mathrm{~ms}$ gave contrasts between $0.15<V<0.2$ on an unresolved calibrator. Knowing that the average measured AMBER instrumental contrast is $V=0.8$ in medium resolution (MR) and $V=0.7$ in low resolution (LR), this yields an actual VLTI+atmosphere contrast of $V_{\text {actual }}=0.12$ in low resolution and $V_{\text {actual }}=0.14$ in medium resolution. If the VLTI was only limited by the atmospheric piston jitter and by vibrations "within the specifications" (i.e. 10\% of contrast loss due to VLTI non atmospheric effects), these contrasts would be $V_{\text {potential }}=0.6$ in medium resolution and $V_{\text {potential }}=0.7$ in low resolution. The commissioning with AT indeed confirmed these VLTI+atmosphere contrast values. Putting these actual and potential contrast values in Eq. (20) yields the number of photons per channel and per frame needed to achieve the condition $\frac{V_{m}^{i j}}{\sigma_{V}} \simeq 1$. These values are given in Table 2 . Table 3 gives the number of photons per frame and per spectral channel collected during the first commissioning of the instrument, before the implementation of the IRIS focal laboratory "infrared image sensor". Combining the collected flux given in Table 3 and the minimum needed flux in Table 2 yields the current and potential limiting magnitudes of AMBER displayed in Table 4. The "current" value corresponds to the currently measured fluxes and atmosphere+VLTI+AMBER instrumental visibility. The "achieved" value gives the magnitude of calibrators on which actual fringes have been recorded. This value is available only for average conditions because of the limited number of attempts. In LR mode there is a gap between the "current" and the "achieved" values. The most likely explanation was the fast fringe drift due to the poor quality of early delay-line models. It is worth 
noting that the current values should allow a successful observation of NGC1068 and maybe of a couple of other AGN (if the MACAO adaptive optics behave correctly on these faint and extended sources in the visible).

The "potential" value corresponds to the currently measured transmissions combined with a VLTI+atmosphere contrast with reduced vibrations allowing the UT to approach the AT vibration levels and the initial VLTI specifications. The limiting magnitude in LR should then be $K \geq 11$, therefore compliant with AMBER specifications for an ambitious AGN program. This limit should be extended to all modes as soon as fringe tracking is operational. The final limit will be set by the quality of the vibration correction. These limiting magnitudes correspond to an $S N R=1$ per spectral channel and per frame. Achieving an accuracy on $V \simeq 0.01$ requires averaging $10^{4} 50 \mathrm{~ms}$ frames, which requires between 500 and 1500 seconds of actual observations.

\subsection{Accuracy on the differential phase}

By analogy with the visibility error computation, the phase uncertainty is written as

$\sigma_{\phi} \simeq \frac{\sigma_{V} / \sqrt{2}}{V_{m}^{i j}}$.

Usually the reference channel is set much larger than the individual spectral channel, and it contributes little to the error on the differential phase, which allows the above estimation to be used for the differential phase. Typically a visibility fundamental SNR of 100 therefore corresponds to a differential phase error of 0.007 radians. However, the differential phase is not affected by the same calibration problems. Currently we approach $10^{-3} \mathrm{rad}$ on very bright sources for which we should have smaller errors. We are currently limited by data-processing problems (problem of the optimum correction of the frame-by-frame piston) and by the difficulty of fitting variable chromatic OPD, mainly due to variations in the thickness of water vapor in the tunnels.

\subsection{Accuracy on the closure phase}

In theory, the accuracy on the closure phase should be about $\sqrt{3} \sigma_{\phi}$. However, with the UT, the number of frames where the three baselines simultaneously show good fringes is extremely small. In practice, the limit is about $0.01 \mathrm{rad}$, even for very bright sources. The situation is supposed to be very different with three AT. The current impossibility of having very accurate closure phases with 3 UT is a serious drawback for the ESP program.

\section{Operating AMBER}

AMBER is working within the standard Science Operations framework in use for the VLT instruments. The VLTI Science Operations is described in Rantakyrö et al. (2004), so here we highlight only those issues relevant to the AMBER general user, who can concentrate on the scientific objectives of the run rather than on details of the telescopes, VLTI, and AMBER operations. In this framework, the user's main, if not single, concern is to make certain that the proper calibration procedures are used.

\subsection{Internal P2VM calibration}

The first calibration is to measure the P2VM specific to the observing mode. The P2VM can be modified by any change in the spectrograph setup, and the operating procedure will force the observer to measure a new P2VM for any new set up, prior to science observations. The observer must choose between a standard-accuracy P2VM, to be within the specifications of AMBER, and a high-accuracy one, to try to approach the goals of highest accuracy.

- Standard-accuracy P2VM: to achieve 0.01 absolute visibility accuracy, a P2VM is computed with about $10^{5}$ photons recorded in each line of each interferogram. With the maximum flux allowed without saturating the detector, a full calibration takes about $7 \mathrm{~min}$, i.e. about the time for the VLTI to acquire the source and put it through all its subsystems. The errors introduced by the standard P2VM are substantially decreased for differential phase (0.001 radians) or visibility (0.001);

- High-accuracy P2VM: In practice, extremely high accuracy $\left(10^{-4} \mathrm{rad}\right)$ is expected only with differential measures and a $10^{7}$-photon P2VM should be sufficient. This needs three hours before the beginning or after the end of the night, so it implies restrictions on the scheduling of setup changes. It still has to be investigated whether the stability of the P2VM over a few hours is good enough to allow the use of such a long calibration.

\subsection{Detector noise and background calibration}

Any set of observations includes observing dark images (warm shutters closed) and sky images (telescopes offset) that are later used in the data reduction. The observer chooses the number of dark and sky exposures. The standard value is currently one dark and one sky for five source exposures. The observers also define the offset of the telescopes, to make sure that the "sky" direction is actually source free.

\subsection{External calibration using a calibrator}

All interferometric observables, including the differential and the closure phases, are affected by systematic effects that can be removed or reduced using a calibrator star with known complex visibility. An important task for the observer is to choose good calibrators. Ideally a calibrator is a point source; however, finding strictly non resolved and bright-enough stars is a real problem. A good calibrator is then a single, non variable star, which can be considered as a uniform disk with a known diameter. Then the visibility of the calibrator is known and its differential and closure phases are zero. "Primary" calibrators, found in databases of measured diameters for bright stars (Richichi et al. 2004), are used when they are close enough to the science target in magnitude, visibility and angular coordinates. The same databases of measured diameters have been used to validate tools for selecting and qualifying much more numerous "secondary" calibrators from the color indices found in astronomical catalogues (Bonneau et al. 2006).

\subsection{Spectral calibration}

A spectral calibrator is required for medium and high spectralresolution modes. Usually, it is a star with a clean and wellknown continuum allowing calibration of the relative intensities recorded by AMBER and use of the telluric lines for accurate wavelength-table calibration. It is also strongly advised to systematically obtain a calibrated high-resolution spectrum of the source during or close to the interferometric run, from ISAAC (Moorwood et al. 1999) or other spectrographs.

\subsection{Beam commutation for closure and differential phase calibration}

To have accurate differential and closure phases, the observer can use the "differential" observing mode, in which a beam 
commutation device (BCD) is used to exchange beams 1 and 2 at the entrance of AMBER at the end of each exposure. This inverts the source differential phase for $B_{12}$ and the source closure phase without changing the instrumental terms. Then, computing the differences between exposures with and without beam commutation (BCD "in" and "out") yields differential and closure phase with strongly reduced instrumental effects (Petrov et al. 2003; Vannier et al. 2006). The commutation takes less than $5 \mathrm{~s}$ and can therefore be performed much more often than a science-calibrator exchange.

\subsection{Observing}

Observing is performed through the VLT control system using the standard tool (P2PP: Phase 2 Preparation Package) to create blocks of observations. These blocks contain several procedures, called templates, to set up the instrument, to point the interferometer, to optimize the beam injection into AMBER, to search for fringes, and to acquire observation data. P2PP allows changing some of the parameters for these procedures, like the wavelength range, the spectral dispersion, or the detector integration time.

The observation block is executed through the breaker of observing blocks (BOB), which ensures that all steps are carried out successfully. Some of the templates are executed in parallel, such as the injection of light into the AMBER fibers for each telescope. The night astronomer has control over BOB and can abort or redo part of the procedures. The system also asks the night astronomer to make critical choices such as whether to continue or abort a so far unsuccessful fringe search.

\subsection{Quick look, online data reduction, archiving}

As for other VLT instruments, AMBER has a standard real-time display that shows the raw data. It also has a quick-look monitor that displays visibilities and phases reduced online in almost real time. The data is automatically archived and passed to the data reduction pipeline that analyses the data almost immediately after acquisition so that the night astronomer and/or the visiting astronomer can check the quality of the data.

\section{Conclusion}

With its coherent combination of three unit telescopes, AMBER currently makes the VLTI the world's largest optical telescope with a total surface $>150 \mathrm{~m}^{2}$ and a maximum baseline $>130 \mathrm{~m}$ corresponding to a spatial resolution than 3 mas in the $K$ band.

The capacity to measure closure phases gives AMBER an image-reconstruction capability with different configurations of three VLTI telescopes. The measurement of individual closure phases already yields decisive constraints on the source geometry. AMBER includes a high-performance infrared-cooled spectrograph allowing the structure and the physics of the source to be constrained by comparing the measures at different wavelengths. Differential interferometry based on simultaneous interferometric measures in different spectral channels yields a gain in accuracy of at least a factor 10. Analyzing spectral channels simultaneously boosts the uniqueness and robustness of measured parameters and images, particularly with spectral regularization functions. At low spectral resolution, the spectral coverage massively increases the instantaneous spatial coverage. AMBER made its first fringes in Paranal in the second quarter of 2004. For the general user, the first 3-UT period started in October 2005 and 3-AT open time is starting in April 2007. Currently it is possible to observe in low, medium, and high resolution in the $H$ and $K$ bands. The full potential in the medium and high spectral resolution depends on the availability of the three-telescope fringe tracker FINITO, which has already been successfully tested with AT and will very likely be available with ATs in October 2007. With the UTs, this depends on the quality of the correction of the vibrations that currently affect the UT Coudé trains.

The first astrophysical observations in 2004 and 2005 has led to a set of refereed papers that can be found in this volume. They all illustrate the power of the spectral dimension of interferometric measures in situations of very poor u-v coverage. On young Herbig stars with high mass (MWC 297; Malbet et al. 2007) and intermediate mass (HD 104237; Tatulli et al. 2007a), the variation of visibility through $\mathrm{Br} \gamma$ allowed them to discuss the connection between dust envelope and wind-driven gas and their relative geometry. On the Be star $\alpha$ Arae (Meilland et al. 2007b), observations in the continuum and in the Bry line allowed severe constraints on a wind found to be axisymmetric with a thin equatorial disk in Keplerian rotation and a very strong polar flow. Another Be star, $\kappa$ CMa (Meilland et al. 2007a), showed a very different, small, non axisymmetric, and probably non-Keplerian envelope. The dusty B[e] star CPD-57 2874 (Domiciano de Souza et al. 2007), observed with AMBER and MIDI, has non-spherical dust and gas envelopes with different global geometries, perhaps again due to polar gas outflows. For the Wolf Rayet $+\mathrm{O}$ binary system $\gamma^{2}$ Velorum (Millour et al. 2007), measures including a strong closure phase in the continuum and in various carbon and helium emission lines, allowed the separation of the components spectra, the measure of the angular separation implying a revision of the Hipparcos distance, and the detection of material outside the immediate vicinity of the components that might be the signature of the wind-wind collision. Measurements in medium and high spectral resolution of the most luminous and massive luminous blue variable $\eta$ Carinae (Weigelt et al. 2007), allowed reconstruction of a central, optically-thick, wind structure elongated in the direction of the axis of the Homonculus nebulae and well-modeled in the continuum and emission lines with remarkable agreement with the radiative transfer code of Hillier \& Lanz (2001). Last but not least, Chesneau et al. (2007) reported the early AMBER interferometric observation of the outburst of RS Oph performed 5.5 days after the outburst which provided an estimation of the extent of the continuum, $\mathrm{Br} \gamma$ and $\mathrm{He}$ I $2.06 \mu \mathrm{m}$ forming regions and some physical constraints on the ejection process. The global picture that emerges is a non-spherical fireball at highvelocity expansion.

The transmission measured on AMBER and the VLTI shows that the current coherent limiting magnitude is $K \simeq 9.0$ with a VLTI+atmosphere fringe contrast of 0.12 . When the correction of the UT vibration allow a VLTI+atmosphere fringe contrast of 0.5 , the limiting coherent magnitude of AMBER on the VLTI will reach $K \simeq 11.5$ in the $20 \%$ best-seeing conditions. This will allow breakthrough science on a large number of AGN and QSO. With the ATs, the VLTI+atmosphere fringe contrast already ranges from 0.5 to 0.9 . When FINITO is operational, all spectral resolution modes will be accessible on the AT for $K \simeq 6$ in average conditions and $K \geq 7$ in the $20 \%$ best conditions, with enormous possibilities in stellar physics.

Acknowledgements. The AMBER project ${ }^{4}$ was founded by the French Centre National de la Recherche Scientifique (CNRS), the Max Planck Institute für Radioastronomie (MPIfR) in Bonn, the Osservatorio Astrofisico di Arcetri (OAA) in Firenze, the French Region Provence Alpes Côte D'Azur,

\footnotetext{
4 The structure and members of the AMBER Consortium can be found in the website: http://amber.obs.ujf-grenoble.fr
} 
and the European Southern Observatory (ESO). The CNRS funding has been made through the Institut National des Sciences de l'Univers (INSU) and its Programmes Nationaux (ASHRA, PNPS, PNP).

The OAA co-authors acknowledge partial support from MIUR grants to the Arcetri Observatory (A LBT interferometric arm, and analysis of VLTI interferometric data and From Stars to Planets: accretion, disk evolution and planet formation) and from INAF grants to the Arcetri Observatory (Stellar and Extragalactic Astrophysics with Optical Interferometry). C. Gil's work was supported in part by the Fundação para a Ciência e a Tecnologia through project POCTI/CTE-AST/55691/2004 from POCTI, with funds from the European program FEDER.

The preparation and interpretation of AMBER observations benefit from the tools developed by the Jean-Marie Mariotti Center for optical interferometry $\mathrm{JMMC}^{5}$ and from the databases of the Centre de Données Stellaires (CDS), and of the Smithsonian/NASA Astrophysics Data System (ADS).

We would like to thank the successive directors of INSU/CNRS, G. Debouzy, F. Casoli, and A.-M. Lagrange, along with the ASHRA president P. Léna and the VLT program manager M. Tarenghi of ESO for their crucial help in setting up and supporting the Consortium. We also thank S. Bensammar, V. Coudé du Foresto, and G. Perrin for their advice in defining the AMBER concept and Consortium.

We are very grateful to the ESO staff in Garching and Paranal for their help in the design and commissioning of AMBER.

The key mechanical design and manufacturing have been provided by the Division Technique de l'INSU and by the mechanical workshops of the Observatoire de Bordeaux, Observatoire de la Côte d'Azur, and Université de Nice.

\section{References}

Akeson, R. L., Swain, M. R., \& Colavita, M. M. 2000, in Interferometry in Optical Astronomy, ed. P. J. Léna \& A. Quirrenbach, Proc. SPIE, 4006, 321 Antonucci, R. R. J. \& Miller, J. S. 1985, ApJ, 297, 621

Barman, T. S., Hauschildt, P. H., \& Allard, F. 2001, ApJ, 556, 885

Bonneau, D., Clausse, J.-M., Delfosse, X., et al. 2006, A\&A, 456, 789

Chesneau, O., Nardetto, N., Millour, F., et al. 2007, A\&A, 464, 119

Colavita, M. M. 1999, PASP, 111, 111

Colavita, M., Akeson, R., Wizinowich, P., et al. 2003, ApJ, 592, L83

Colavita, M. M., Wallace, J. K., Hines, B. E., et al. 1999, ApJ, 510, 505

Coudé Du Foresto, V., Perrin, G., Ruilier, C., et al. 1998, in Astronomical Interferometry, ed. R. D. Reasenberg, Proc. SPIE V, 3350, 856

Coudé du Foresto, V., Bordé, P. J., Mérand, A., et al. 2003, in Interferometry for Optical Astronomy II, ed. W. A. Traub, Proc. SPIE, 4838, 280

Delplancke, F., Nijenhuis, J., de Man, H., et al. 2004, in New Frontiers in Stellar Interferometry, ed. W. A. Traub, Proc. SPIE, 5491, 1528

Domiciano de Souza, A., Kervella, P., Jankov, S., et al. 2005, A\&A, 442, 567

Domiciano de Souza, A., Driebe, T., Chesneau, O., et al. 2007, A\&A, 464, 81

Gai, M., Menardi, S., Cesare, S., et al. 2004, in New Frontiers in Stellar Interferometry, ed. W. A. Traub, Proc. SPIE 5491, 528

Glindemann, A., Albertsen, M., Andolfato, L., et al. 2004, in New Frontiers in Stellar Interferometry, ed. W. A. Traub, Proc. SPIE, 5491, 447

Glindemann, A., Ballester, P., Bauvir, B., et al. 2001a, The Messenger, 106, 1

Glindemann, A., Bauvir, B., Delplancke, F., et al. 2001b, The Messenger, 104, 2

Hillier, D. J. \& Lanz, T. 2001, in Spectroscopic Challenges of Photoionized Plasmas, ed. G. Ferland \& D. W. Savin, ASP Conf. Ser., 247, 343

Jaffe, W., Meisenheimer, K., Röttgering, H. J. A., et al. 2004, Nature, 429, 47

Jankov, S., Vakili, F., Domiciano de Souza, Jr., A., \& Janot-Pacheco, E. 2002, in IAU Coll. 185: Radial and Nonradial Pulsationsn as Probes of Stellar Physics, ed. C. Aerts, T. R. Bedding, \& J. Christensen-Dalsgaard, ASP Conf. Ser., 259, 172

Kervella, P., Gitton, P. B., Ségransan, D., et al. 2003, in Interferometry for Optical Astronomy II, ed. W. A. Traub, Proc SPIE, 4838, 858

Lawson, P. R., Cotton, W. D., Hummel, C. A., et al. 2006, in Advances in Stellar Interferometry, ed. J. Monnier, M. Schöller, \& W. C. Danchi, Proc. SPIE, 6268

Leinert, C. 2004, in New Frontiers in Stellar Interferometry, ed. W. A. Traub, Proc SPIE, 5491, 19

Leinert, C., Graser, U., Przygodda, F., et al. 2003, Ap\&SS, 286, 73

Malbet, F. 2003, Ap\&SS, 286, 131

Malbet, F., Benisty, M., De Wit, W. J., et al. 2007, A\&A, 464, 43

Marconi, A., Maiolino, R., \& Petrov, R. G. 2003, Ap\&SS, 286, 245

Mège, P., Malbet, F., \& Chelli, A. 2003, in Interferometry for Optical Astronomy II, ed. W. A. Traub, 329, Proc. SPIE, 4838,

Meilland, A., Millour, F., Stee, P., et al. 2007a, A\&A, 464, 73

5 The JMMC is a center providing software tools for optical interferometry described on the website: http://www. jmmc. fr
Meilland, A., Stee, P., Vannier, M., et al. 2007b, A\&A, 464, 59

Millan-Gabet, R., Malbet, F., Akeson, R., et al. 2007, Protostars and Planets V, 539

Millour, S., Petrov, R., Chesneau, O., et al. 2007, A\&A, 464, 107

Moorwood, A., Cuby, J.-G., Ballester, P., et al. 1999, The Messenger, 95, 1

Mourard, D., Abe, L., Domiciano, A., et al. 2003, in Interferometry for Optical Astronomy II, ed. W. A. Traub, Proc. SPIE, 4838, 9

Paresce, F., Delplancke, F., Derie, F., et al. 2003a, in Interferometry for Optical Astronomy II, ed. W. A. Traub, Proc. SPIE, 4838, 486

Paresce, F., Mourard, D., Bedding, T., et al. 1996, The Messenger, 83, 14

Paresce, F., Van Boekel, R., Correia, S., et al. 2003b, in Interferometry for Optical Astronomy II, ed. W. A. Traub, Proc SPIE, 4838, 235

Petrov, R., Roddier, F., \& Aime, C. 1986, Opt. Soc. Am. J. A: Optics and Image Science 3, 634

Petrov, R. G. 1988, in NOAO-ESO Conference on High-Resolution Imaging by Interferometry: Ground-Based Interferometry at Visible and Infrared Wavelengths, ed. F. Merkle, 235

Petrov, R. G. 1989, in Diffraction-Limited Imaging with Very Large Telescopes, ed. D. M. Alloin \& J.-M. Mariotti, NATO ASIC Proc., 274, 249

Petrov, R. G., Vannier, M., Lopez, B., et al. 2003, in EAS Publ. Series, ed. C. Aime \& R. Soummer, 297

Rantakyrö, F. T., Galliano, E., Hummel, C. A., et al. 2004, in New Frontiers in Stellar Interferometry, ed. W. A. Traub, Proc. SPIE, 5491, 1690

Richichi, A., Percheron, I., \& Khristoforova, M. 2004, VizieR Online Data Catalog, 343, 10773

Robbe-Dubois, S., Lagarde, S., Antonelli, P., et al. 2007, A\&A, 464, 13

Ségransan, D., Beuzit, J.-L., Delfosse, X., et al. 2000, in Interferometry in Optical Astronomy, ed. P. J. Léna \& A. Quirrenbach, Proc. SPIE, 4006, 269

Swain, M., Vasisht, G., Akeson, R., et al. 2003, ApJ, 596, L163

Tatulli, E. \& LeBouquin, J.-B. 2006, MNRAS, 368, 1159

Tatulli, E., Isella, A., Natta, A., et al. 2007a, A\&A, 464, 55

Tatulli, E., Millour, F., Chelli, A., et al. 2007b, A\&A, 464, 29

van Boekel, R., Kervella, P., Schöller, M., et al. 2003, A\&A, 410, L37

Vannier, M., Petrov, R. G., Lopez, B., \& Millour, F. 2006, MNRAS, 367, 825

Weigelt, G., Kraus, S., Driebe, T., et al. 2007, A\&A, 464, 87

Wittkowski, M., Kervella, P., Arsenault, R., et al. 2004, A\&A, 418, L39

1 Laboratoire Universitaire d'Astrophysique de Nice, UMR 6525 Université de Nice, Sophia Antipolis/CNRS, Parc Valrose, 06108 Nice Cedex 2, France, e-mail: Romain.Petrov@unice.fr

${ }^{2}$ Laboratoire d'Astrophysique de Grenoble, UMR 5571 Université Joseph Fourier/CNRS, BP 53, 38041 Grenoble Cedex 9, France e-mail: Fabien.Malbet@obs.ujf-grenoble.fr

3 Max-Planck-Institut für Radioastronomie, Auf dem Hügel 69, 53121 Bonn, Germany

${ }^{4}$ Laboratoire Gemini, UMR 6203 Observatoire de la Côte d'Azur/CNRS, BP 4229, 06304 Nice Cedex 4, France

5 INAF-Osservatorio Astrofisico di Arcetri, Istituto Nazionale di Astrofisica, Largo E. Fermi 5, 50125 Firenze, Italy

${ }^{6}$ European Southern Observatory, Casilla 19001, Santiago 19, Chile

7 ONERA/DOTA, 29 av de la Division Leclerc, BP 72, 92322 Chatillon Cedex, France

8 Centre de Recherche Astronomique de Lyon, UMR 5574 Université Claude Bernard/CNRS, 9 avenue Charles André, 69561 Saint Genis Laval Cedex, France

9 Division Technique INSU/CNRS UPS 855, 1 place Aristide Briand, 92195 Meudon Cedex, France

10 IRCOM, UMR 6615 Université de Limoges/CNRS, 123 avenue Albert Thomas, 87060 Limoges Cedex, France

11 European Southern Observatory, Karl Schwarzschild Strasse 2, 85748 Garching, Germany

12 Kiepenheuer Institut für Sonnenphysik, Schöneckstr. 6, 79104 Freiburg, Germany

13 Instituut voor Sterrenkunde, KU-Leuven, Celestijnenlaan 200D, 3001 Leuven, Belgium

14 Centro de Astrofísica da Universidade do Porto, Rua das Estrelas 4150-762 Porto, Portugal

15 Laboratoire Astrophysique de Toulouse, UMR 5572 Université Paul Sabatier/CNRS, BP 826, 65008 Tarbes Cedex, France

16 Departamento de Astronomia, Universidad de Chile, Chile 